\title{
Perfil e indicadores da assistência de um serviço de atenção domiciliar em cuidados paliativos de um Hospital de Câncer
}

\author{
Service profile description and indicators of a home \\ based palliative care team of a Cancer Hospital in \\ Brasil
}

Luís Fernando Rodrigues ${ }^{1}$, Landina Silene Pacheco ${ }^{2}$, Eliane Fazuoli Chubaci ${ }^{3}$, Gabriela Vieria Zabeu ${ }^{4}$, Graziely de Lima Souza ${ }^{5}$, Isabela Caroline de Freitas Colombino ${ }^{6}$, Sarita Nasbine Frasseto ${ }^{7}$, Alexandre Venâncio de Souza ${ }^{8}$

\footnotetext{
1. Médico, Serviço de Atendimento Domiciliar. Unidade de Cuidados Paliativos Hospital de Câncer de Barretos. Barretos - São Paulo - Brasil. ORCID ID:0000-0001-6177-3769. E-mail: lufe.luis@gmail.com

2. Enfermeira, Serviço de Atendimento Domiciliar. Unidade de Cuidados Paliativos. Hospital de Câncer de Barretos. Barretos - São Paulo - Brasil. ORCID ID: 0000-0001-9444-091X. E-mail: landinapacheco@ hotmail.com

3. Fisioterapeuta, Serviço de Atendimento Domiciliar. Unidade de Cuidados Paliativos. Hospital de Câncer de Barretos. Barretos - São Paulo - Brasil. ORCID ID:0000-0003-3968-3411. E-mail: li_chubaci@ hotmail.com

4. Fisioterapeuta, Serviço de Atendimento Domiciliar. Unidade de Cuidados Paliativos. Hospital de Câncer de Barretos. Barretos - São Paulo - Brasil. ORCID ID:0000-0002-0310-2366. E-mail: gabi_zabeu@ hotmail.com

5. Assistente Social, Serviço de Atendimento Domiciliar. Unidade de Cuidados Paliativos. Hospital de Câncer de Barretos. Barretos - São Paulo - Brasil. ORCID ID:0000-0002-0923-1142. E-mail: grazilimachado1@hotmail.com

6. Fisioterapeuta, Serviço de Atendimento Domiciliar. Unidade de Cuidados Paliativos. Hospital de Câncer de Barretos. Barretos - São Paulo - Brasil. ORCID ID: 0000-0001-7993-6837. E-mail: isabelacolombino@hotmail.com

7. Médica, Serviço de Atendimento Domiciliar. Unidade de Cuidados Paliativos. Hospital de Câncer de Barretos. Barretos - São Paulo - Brasil. ORCID ID: 0000-0001-5378-1793. E-mail: sanafra@gmail.com

8. Psicólogo, Serviço de Atendimento Domiciliar. Unidade de Cuidados Paliativos. Hospital de Câncer de Barretos. Barretos - São Paulo - Brasil. ORCID ID: 0000-0002-1189-5781. E-mail: alexandre. venancio@hotmail.com
}

CONTATO: Luís Fernando Rodrigues | Endereço: Av. 47, nº 0540, entre 14 e 16 - Celina | Barretos | São Paulo | CEP 14780-755 | Telefone: (17) 3312- 9747/ (17) 99105-2649 | E-mail: lufe.luis@ gmail.com 
COMo CITAR: Rodrigues LF, Pacheco LS, Chubaci EF, Zabeu GV, Souza GL, Colombino ICF, Frasseto SN, Souza AV. Perfil e indicadores da assistência de um serviço de atenção domiciliar em cuidados paliativos de um hospital de câncer. R. Saúde Públ. Paraná. 2020 Jul;3(1):05-17.

RESUMO O objetivo deste trabalho é descrever o perfil de um serviço de atendimento domiciliar em cuidados paliativos e os resultados obtidos após o primeiro ano de funcionamento. Foi realizado um estudo transversal, observacional retrospectivo e foram analisados os dados referentes aos atendimentos realizados pelo Serviço de Atendimento Domiciliar em Cuidados Paliativos (SAD/CP) no primeiro ano de funcionamento. Observou-se que, de 72 pacientes elegíveis, 56,9\% eram mulheres e a idade média foi de 67,74 anos. O Karnofsky Performance Status médio foi de 48,16 e o Índice de Prognóstico Paliativo médio foi de 3,23. A maioria dos pacientes faleceu na Unidade de Cuidados Paliativos (51,4\%). Concluiu-se, assim, que os pacientes que morreram em casa receberam mais visitas da equipe de atendimento domiciliar e se deslocaram menos vezes para a urgência e emergência. Fadiga, dor e anorexia foram os sintomas mais frequentes. Ansiedade, depressão e sensação de bem-estar reduzido foram os sintomas mais intensos.

PALAVRAS-CHAVE: Cuidados Paliativos. Institutos de Câncer. Visita Domiciliar. Pessoal Técnico de Saúde. Indicadores Básicos de saúde. Sinais e Sintomas.

ABSTRACT The aim of this study is to describe the profile of a home-based palliative care service (HBPCS) and the results after the first year of work. A cross-sectional observational retrospective study was conducted and data about the visits made by the home-based palliative care team were analyzed regarding the first year of work. From data of 72 elegible patients, 56,9\% were women and the average age was 67.7 years. The average Karnosfsky Performance Status was 48.16, and the average Palliative Prognostic Index was 3,23. The majority of the patients died at the Palliative Care Unit (51.4\%). The patients who died at home received more visits of HBPCS and had less visits to the emergency department. Fatigue, Pain and Anorexia were the most common symptoms. Anxiety, Depression and reduced Well-Being sensation were the most intense symptoms.

KEYWORDS: Palliative Care. Cancer Care Facilities. House Calls. Allied Health Personnel. Health Status Indicators. signs and Symptoms. 


\section{INTRODUÇÃO}

A

qualidade da organização dos cuidados pode ser avaliada de forma válida e confiável usando indicadores de qualidade de processos e estrutura'. O Atendimento Domiciliar é um dos modelos de atenção à saúde em que os Cuidados Paliativos podem ser enquadrados². A literatura mundial é pródiga em mostrar que o local preferido das pessoas para receberem cuidados em final de vida é o domicílio. No entanto, existe desacordo entre o lugar declarado como preferido e o lugar em que de fato o paciente é cuidado e venha a morrer ${ }^{3-7}$. Existem estudos sugerindo que o óbito no domicílio seria um indicador de qualidade de serviços paliativos prestados ${ }^{8.9} \mathrm{e}$ essa probabilidade aumenta quando o paciente recebe visitas de equipes especializadas de cuidados paliativos e com frequência alta ${ }^{10}$. Porém, é preciso ter cautela para se usar esse indicador como parâmetro de qualidade para serviços de atendimento domiciliar, pois a morte em casa pode trazer carga adicional de cuidados e distress para a família e/ou cuidadores"1. Uma revisão sistemática brasileira apontou que essa sobrecarga se dá nos planos físico, psíquico e social'². No Brasil, os óbitos acontecem com maior frequência nos hospitais ${ }^{13.14}$. Parece existir evidência de que pacientes com câncer avançado, sob cuidados paliativos prestados por equipe especializada, têm sobrevida aumentada comparados com grupo de pacientes que recebeu cuidados usuais ${ }^{15}$.

No Brasil, a política pública que regulamenta e cria fonte de financiamento para o atendimento domiciliar é o Programa Melhor em Casa, lançado em 2011. Nele está prevista a oferta de cuidados paliativos, inclusive com a possibilidade de que a morte ocorra em casa, sugerindo inclusive um indicador para esse fenômeno ${ }^{16}$ Além desse, uma lista de 18 indicadores foi proposta para avaliação de serviços de atendimento domiciliar em geral, sem a especificação dos Cuidados Paliativos ${ }^{17.18}$.

O Caderno 2 do Programa Melhor em Casa ${ }^{16}$, que trata da inserção dos Cuidados Paliativos, não sugere ou determina indicadores de desempenho específico para esse agravo/condição, conforme denominado no Manual Instrutivo de Monitoramento e Avaliação.

Não existe ainda consenso sobre grupos de indicadores a serem utilizados para avaliar serviços de cuidados paliativos e vários autores têm tentado elaborar um conjunto de parâmetros que sejam mais factíveis e confiáveis para serem empregados 1.19-23.

Portanto, a forma e os instrumentos escolhidos para avaliar a qualidade do desempenho de serviços variam de acordo com a cultura e o país e não existe consenso ou benchmarks testados que permitam padronizar formas de avaliação dos serviços, embora o estudo europeu de 2014 tenha evoluído no sentido de reduzir o número de indicadores empregados para avaliação de qualidade ${ }^{19}$. Também parece haver semelhanças entre os estudos disponiveis referentes aos parâmetros utilizados para avaliação de qualidade dos serviços, quais sejam: os aspectos físicos do paciente, aspectos psíquicos e emocionais dos pacientes e familiares, avaliação de recursos disponíveis para o cuidado e aspectos espirituais.

Não existem, até o momento, iniciativas brasileiras que visem avaliar o desempenho de serviços e contextos de Cuidados Paliativos, levando em consideração as diferenças entre esses serviços. Além disso, o Programa Melhor em Casa considera Cuidados Paliativos como um dos componentes de "agravos/condição" e não estabelece indicadores específicos para essa população. Portanto, este estudo tem por objetivo geral realizar a descrição do perfil de um serviço de atendimento domiciliar em cuidados paliativos de hospital de câncer. Os objetivos específicos foram:

1) Descrever a estrutura dos indicadores de processos utilizados no Serviço de Atendimento Domiciliar 
em Cuidados Paliativos (SADP) de um hospital especializado em tratamento de câncer; 2) analisar os tempos decorridos entre distintos momentos do tratamento dos pacientes após a admissão em unidade de cuidados paliativos; 3) analisar outras variáveis como média de permanência, frequência de óbitos, frequência de visitas ao serviço ambulatorial de urgência e emergência do hospital de câncer, sintomas prevalentes iniciais, o Karnofsky performance status (ou Escala de Desempenho de Karnofsky) e o índice de prognóstico paliativo na primeira visita, entre outros.

Para avaliar a prevalência e intensidade dos sintomas, foi utilizada a escala ESAS (Edmonton Symptom Assessment Scale ou Escala de Avaliação de Sintomas de Edmonton). É uma escala composta por 9 sintomas a serem avaliados: dor, náusea e vômito, fadiga, depressão, ansiedade, sonolência, apetite, dispneia e bemestar geral. Esses sintomas são classificados numericamente pelo paciente numa gradação que vai de 0 (zero), que significa ausência de sintomas, até 10 (dez), pior sintoma possível, com intervalos de 1 unidade.

Para avaliar estado de desempenho ou performance status, foi utilizada a escala de Karnofsky. É uma escala de 10 níveis, na qual o valor 0 (zero) é atribuído ao paciente morto e 100 (cem) ao paciente com plena atividade e sem sintomas de doença, com intervalos de 10 em 10.

A escala PPI (Palliative Prognostic Index ou Índice de Prognóstico Paliativo) é uma escala composta por 5 parâmetros principais: valor do KPS (que varia de 0 a 100), ingestão oral (normal recebe nota 0 , moderadamente reduzida recebe nota 1 e gravemente reduzida recebe nota 2,5), presença de edema periférico (ausente recebe nota 0 , presente nota 1), presença de dispneia de repouso (ausente nota 0, presente nota 3,5) e presença de delirium (ausente nota 0, presente nota 4,5). Faz-se o somatório e, de acordo com o valor, é possivel fazer uma estimativa de sobrevida que varia de menos de 3 semanas a mais de 6 semanas.

O uso dessas escalas é rotineiro e sua importância reside no fato de ser o ponto de partida para a avaliação dos pacientes e, consequentemente, a elaboração do plano terapêutico antecipado individualizado.

\section{MÉTODO}

Este artigo é resultado de um estudo observacional e analítico transversal, retrospectivo. Foram coletados dados já registrados em prontuário dos pacientes atendidos no SADP de um hospital de câncer.

Os dados foram coletados dos registros de prontuários de pacientes atendidos em um hospital de câncer do interior do estado de São Paulo. Na cidade, esse hospital possui três unidades, sendo uma delas a Unidade de Cuidados Paliativos, também referido como unidade 2 e distante $4 \mathrm{~km}$ da unidade principal (Unidade 1). A unidade 3 é o hospital infantojuvenil. A unidade de cuidados paliativos (Unidade 2) conta com 50 leitos de internação, 8 de observação, ambulatório e uma central de intercorrência ambulatorial paliativo, de uso exclusivo para pacientes do hospital que apresentem crises de sintomas. Esta unidade tem $80 \%$ de 41 profissionais de nível superior treinados em cuidados paliativos em nível de especialização e possui setor de educação corporativa para treinamento e atualização dos profissionais de nível médio. Desde 2014. conta com o Serviço de Atendimento Domiciliar Paliativos (SADP) baseado no Hospital São Judas que é a Unidade 2, composto por uma enfermeira (tempo integral), uma técnica de enfermagem (tempo integral), um motorista (tempo integral), um médico (tempo parcial), uma psicóloga (tempo parcial), um fisioterapeuta (tempo parcial) e uma assistente social (tempo parcial). Os profissionais descritos como tempo parcial dedicam parte do seu tempo de contrato para o SADP e parte para atividades em outros setores da Unidade de Cuidados Paliativos. Caso algum paciente precise da avaliação e intervenção de nutricionista, fonoaudióloga, 
dentista, terapeuta ocupacional ou musicoterapeuta, é possível requisitá-los com antecedência para que o atendimento seja agendado. Esses profissionais são integrantes da Unidade de Cuidados Paliativos.

A equipe dispõe de 1 automóvel do tipo minivan com capacidade para sete pessoas, onde são transportados os membros da equipe e os equipamentos e materiais médico-hospitalares.

As visitas são realizadas de segunda a quinta, no período das 7h às 17h com intervalo para almoço. Essas visitas são realizadas predominantemente pela enfermeira e pela técnica de enfermagem, que se encarregam de elaborar a agenda de atendimento e identificar as demandas para os outros profissionais. A sexta-feira é o dia dedicado à reunião interdisciplinar que tem a participação de todos os membros da equipe e dura em média 1 hora e meia. Também é o dia dedicado às atividades administrativas (elaboração de agenda, reposição de materiais, alimentação de planilhas e manutenção do veículo).

A área de abrangência de atendimento corresponde a 18 municípios que compõem o Departamento Regional de Saúde onde o hospital está localizado, com uma extensão de $8.098,60 \mathrm{~km}^{2}$ e uma população de 432.830 habitantes. 0 município mais próximo da cidade-sede fica a $16 \mathrm{~km}$ e o mais distante, a $94 \mathrm{~km}$. A distância entre os municípios situados nos extremos norte e sul da região é de aproximadamente $134 \mathrm{~km}$ e a maior distância entre os municípios localizados nos extremos leste e oeste, é de 86 km.

Os pacientes admitidos no SADP são provenientes exclusivamente do Hospital de Câncer, encaminhados para a Unidade de Cuidados Paliativos (UCP). Então, são avaliados e, caso tenham critérios de elegibilidade preenchidos, são encaminhados ao SADP. Os critérios para elegibilidade são:

1) Desejo e/ou permissão por parte do paciente e/ou familiares para receber o atendimento domiciliar:

2) ter diagnóstico de câncer em estágio avançado;

3) ter passado por pelo menos uma consulta ou internação na Unidade de Cuidados Paliativos;

4) ter um Karnofsky performance status medido no mínimo de 50\%;

5) residir na área de abrangência da Regional de Saúde do município-sede;

6) residir em habitação com condições mínimas de serviço de água e luz;

7) ter um ou mais cuidadores aptos, que podem estar próximos ao paciente 24 horas;

Em caso de crises, os pacientes e familiares são orientados a recorrer a um aplicativo de comunicação popular para mandar mensagens e/ou fazer ligação para o celular da equipe. Após o horário de funcionamento, a recomendação é para que liguem na Unidade de Cuidados Paliativos (UCP) e falem com uma das enfermeiras de plantão ou o médico do plantão.

Caso a crise não se resolva, ou se for opção da família, o paciente pode ser trazido à Central de Intercorrência Ambulatorial (CIAP) da UCP. O transporte é feito por veículo próprio, SAMU ou ambulância do município.

Para descrição dos indicadores, foram considerados os registros completos de todos os pacientes admitidos no SADP de 11 de agosto de 2014 a 10 de agosto de 2015. Foram considerados elegíveis para este estudo os registros completos dos pacientes na primeira visita e excluídos os registros considerados incompletos na primeira visita.

Os dados coletados foram: 1) tempo transcorrido entre o diagnóstico de câncer e o encaminhamento para a unidade de cuidados paliativos; 2) tempo transcorrido entre o recebimento do encaminhamento para o SADP e a visita de admissão medido em dias; 3) média de permanência geral (corresponde ao indicador 1.1 do Programa Melhor em Casa (PMC) - refere-se ao tempo transcorrido entre a admissão no SAD/CP e o 
óbito; 4) perfil de sintomas na primeira visita no domicílio, de acordo com a Escala de Avaliação de Sintomas de Edmonton (ESAS) 24.25 ; 5) perfil de performance status na primeira visita no domicílio, de acordo com a Escala de Desempenho de Karnofsky (KPS ${ }^{26}$; 6) prognóstico de sobrevida na primeira visita no domicilio, de acordo com o Índice de Prognóstico Paliativo (PPI)27.28; 7) frequência de óbitos no domicílio; 8) frequência de visitas à Central de Intercorrência Ambulatorial Paliativo (CIAP), que corresponde ao indicador 10 do PMC "procura por serviço de urgência"; 9) número de pacientes atendidos, que corresponde ao indicador 6 do PMC "capacidade total de usuários acompanhados/mês na AD; 10) número médio de visitas por paciente (indicador 14 do PMC); 11) média de PPI na primeira visita; 12) sintomas prevalentes na primeira visita; 13) média de KPS na admissão; 14) média de ESAS por sintoma na admissão. Além disso, foram coletados dados referentes a sexo, idade, cidade de procedência, estado de procedência, bem como dados clínicos referentes ao câncer (sítio primário do tumor, sítio das metástases).

Como base para a elaboração do conjunto de dados coletados neste estudo, utilizou-se os modelos constantes no manual do Programa Melhor em Casa, do Ministério da Saúde ${ }^{29}$, e o modelo do Serviço de Atendimento Domiciliar, da Universidade Federal de Pelotas ${ }^{30}$. 0 indicador "frequência de visitas à Central de Intercorrência Ambulatorial" que equivale ao indicador "Procura pelo setor de urgência e emergência", além de fazer parte dos indicadores sugeridos pelo Programa Melhor em Casa, também compõe o conjunto de indicadores de qualidade em final de vida definido previamente em outros estudos ${ }^{31,32}$.

Os dados foram obtidos mediante consulta aos prontuários dos pacientes atendidos pelo SADP. Solicitou-se dispensa do uso do Termo de Consentimento Livre e Esclarecido (TCLE), considerando que este estudo não envolve diretamente os pacientes, que os dados são de registro rotineiro da equipe e têm caráter administrativo, que o objetivo do estudo não é testar nenhum instrumento novo e nem validar nenhum instrumento, que não se está submetendo o paciente a teste de drogas ou procedimentos, que os riscos ao paciente são mínimos, que o risco de perda de sigilo das informações é inerente a qualquer pesquisa e que os autores se comprometem a providenciar todas as precauções necessárias para proteger os dados.

O projeto foi submetido à apreciação da Comissão de Ética em Pesquisa (CEP). Foram utilizadas as determinações da Resolução N 466/2012, do Conselho Nacional de Saúde (CNS) e foi aprovado pelo CEP. sob número 1163/2016 - 04/08/2016 (CAEE: 57205015.2.0000.5437).

Foi utilizada a descrição simples de frequências e porcentagens, usando tabelas e gráficos. Foi aplicado o teste de Kruskal Wallis a fim de avaliar se houve influência entre o número de visitas domiciliares recebidas e as frequências de procura pela urgência/emergência e óbitos no domicílio. Também foi analisado se houve interferência das variáveis KPS, PPI, idade, sexo, estadiamento clínico ou número de metástases sobre os tempos medidos ou local de óbito, dos pacientes atendidos pelo SADP.

\section{RESULTADOS}

Entre 11 de agosto de 2014 a 10 de agosto de 2015, 76 pacientes foram admitidos no Serviço de Atendimento Domiciliar (SADP), dos quais 72 registros foram considerados satisfatórios para compor a análise. Quatro registros foram excluídos por não conterem minimamente as informações exploradas neste estudo. Dos 72 registros avaliados, 41 (56,9\%) eram mulheres, 93\% oriundos de municípios da DRS de abrangência e $94,4 \%$ do estado de São Paulo. A média das idades e outras variáveis descritivas estão mostradas na Tabela 1. 
Tabela 1. Variáveis descritivas na população estudada, SADP, Hospital de Câncer de Barretos, 2014-2015.

\begin{tabular}{|c|c|c|c|c|c|c|}
\hline & $\begin{array}{c}\mathbf{N} \\
\text { (pacientes) }\end{array}$ & MÉDIA & DP & MEDIANA & Min & MÁx \\
\hline IDADE NA ADMISSÃO & 69 & 67,74 & 15,88 & 72,57 & 28,57 & 93,64 \\
\hline KPS & 38 & 48,16 & 12,27 & 40,00 & 20,00 & 70,00 \\
\hline PPI & 33 & 3,23 & 2,14 & 2,50 & 0,00 & 8,50 \\
\hline $\mathrm{N}^{\circ}$. de visitas do SADP recebidas & 68 & 5,13 & 4,24 & 3,50 & 1,00 & 20,00 \\
\hline $\mathrm{N}^{\circ}$ de idas ao ambulatório (CIAP) & 69 & 2,19 & 2,63 & 1,00 & 0,00 & 16,00 \\
\hline $\begin{array}{l}\text { Tempo (em dias) entre a entrada na U1 } \\
\text { até a entrada na UCP }\end{array}$ & 66 & 1992,65 & 2495,25 & 654,50 & 1,00 & 9241,00 \\
\hline $\begin{array}{l}\text { Tempo (em dias) entre a entrada na U2 e } \\
\text { encaminhamento para o SADP }\end{array}$ & 45 & 219,64 & 452,86 & 5,00 & 0.00 & 1861,00 \\
\hline $\begin{array}{l}\text { Tempo entre encaminhamento e aceite } \\
\text { no SADP (em dias) }\end{array}$ & 44 & 13,86 & 15,49 & 8,00 & 0,00 & 74,00 \\
\hline Tempo de sobrevida no SADP (em dias) & 51 & 80,63 & 102,03 & 50,00 & 1,00 & 541,00 \\
\hline $\begin{array}{l}\text { Tempo no SADP até internação na UCP } \\
\text { com óbito (em dias) }\end{array}$ & 37 & 62,08 & 93,45 & 37,00 & 1,00 & 527,00 \\
\hline $\begin{array}{l}\text { Tempo de internação na UCP até o óbito } \\
\text { (em dias) }\end{array}$ & 37 & 8,78 & 9,51 & 5,00 & 0.00 & 44,00 \\
\hline
\end{tabular}

Fonte: os autores (2015)

A maioria dos pacientes atendidos no primeiro ano do programa tinha diagnóstico de câncer, sendo que 56 (91,8\%) tinham câncer em estadiamento clínico III ou IV na admissão no SADP. Os locais mais frequentes de metástases foram, por ordem de frequência, ossos 16 (27,1\%), pulmão 14 (23,3\%) e fígado 13 (21,7\%). Dos 72 casos avaliados, $50(69,4 \%)$ pacientes haviam falecido até o término do estudo, sendo que $37(51,4 \%)$ morreram na UCP, 7 (9,7\%) morreram no domicílio sob os cuidados do SADP e 6 (8,3\%) no hospital da sua cidade de origem. Dezesseis pacientes estavam vivos e não havia informação disponível para $6(8,3 \%)$ pacientes.

A fim de verificar se os tempos medidos (de encaminhamento da unidade 1 para a UCP, da unidade 2 para o SADP, sobrevida nos SADPs e tempo de hospitalização na última internação que levou ao óbito) ou o local de óbito sofreram influência das variáveis clínicas ou dos locais de origem do paciente, estas foram testadas estatisticamente. Após análise, não se observou interferência do KPS, PPI, idade, sexo, estadiamento clínico ou número de metástases sobre os tempos medidos ou o local de óbito. No tempo de sobrevida após a entrada no SADP, houve diferença estatística significante no tempo de sobrevida de pacientes com PPI > 4 e KPS < 40. Os pacientes com KPS > 40 tiveram uma sobrevida de 85,1 dias em média (DP=43,5; mediana de 76,0 dias), enquanto que os pacientes com KPS $<40$ tiveram uma sobrevida média de 42,8 dias (DP=61,9; mediana de 23,5 dias; $[p=0,003]$ ).

Os pacientes com PPI > 4 (sinal de sobrevida menor de 6 semanas) tiveram sobrevida média de 16,2 dias ( $\mathrm{DP}=14,5$; com mediana de 10 dias), enquanto os pacientes com $\mathrm{PPI}<4$ tiveram sobrevida média de 63,5 dias ( $\mathrm{DP}=43,4$; mediana de 57,5 dias), com diferença estatística significante $(p=0,004)$. Com relação ao local 
de óbito, a maioria dos pacientes (51,4\%) faleceu na Unidade de Cuidados Paliativos. Os dados encontram-se na Tabela 2.

Tabela 2. Distribuição de acordo com local de óbito - SADP, Hospital de Câncer de Barretos, 2014-2015.

\begin{tabular}{|ccc}
\hline LOCAL DE ÓBITO & $\mathbf{N}$ & $\%$ \\
\hline UCP* & 37 & $51,4 \%$ \\
\hline Domicílio com SADP & 7 & $9,7 \%$ \\
\hline Hospital da origem & 6 & $8,3 \%$ \\
\hline Não se aplica (vivos) & 16 & $22 \%$ \\
\hline Status ignorado & 6 & $8,3 \%$ \\
\hline
\end{tabular}

*UCP - Unidade de Cuidados Paliativos

Fonte: os autores (2015)

Procurou-se saber se o número de visitas recebidas pelos pacientes poderia ter influência sobre o local de óbito e se o número de visitas ao setor de Urgência da UCP influenciaria o local do óbito, o que não se comprovou conforme os dados expostos na Tabela 3.

Os sintomas que mais prevaleceram em termos de frequência na primeira visita (visita de admissão ou visita de inclusão) estão descritos no Gráfico 1.

Tabela 3. Distribuição dos locais e óbito de acordo com frequência de visitas programadas e idas ao Setor de Urgência/Emergência (U/E), SADP, Hospital de Câncer de Barretos, 2014-2015.

\begin{tabular}{lcccc}
\hline & \multicolumn{3}{c}{ LOCAL DE ÓBITO } & VALOR DE $\boldsymbol{p}$ \\
\cline { 2 - 4 } VARIÁVEIS & Domicilio & UCP & $\begin{array}{c}\text { Hospital } \\
\text { Origem }\end{array}$ & VA \\
\cline { 2 - 4 } & 1,29 & 2,68 & 2,50 & 0,368 \\
\hline Idas ao setor de U/E & 6,57 & 3,84 & 4,83 & 0,390 \\
\hline
\end{tabular}

Fonte: os autores (2015)

Se levarmos em conta a intensidade dos sintomas, a distribuição ocorrerá conforme demonstrado no Gráfico 2, no qual ansiedade e depressão foram os sintomas de maior intensidade média, conforme medidos pela Escala de Avaliação de Sintomas de Edmonton (ESAS). 
Gráfico 1. Prevalência dos Sintomas de acordo com a frequência, SADP, Hospital de Câncer de Barretos, 2014-2015.

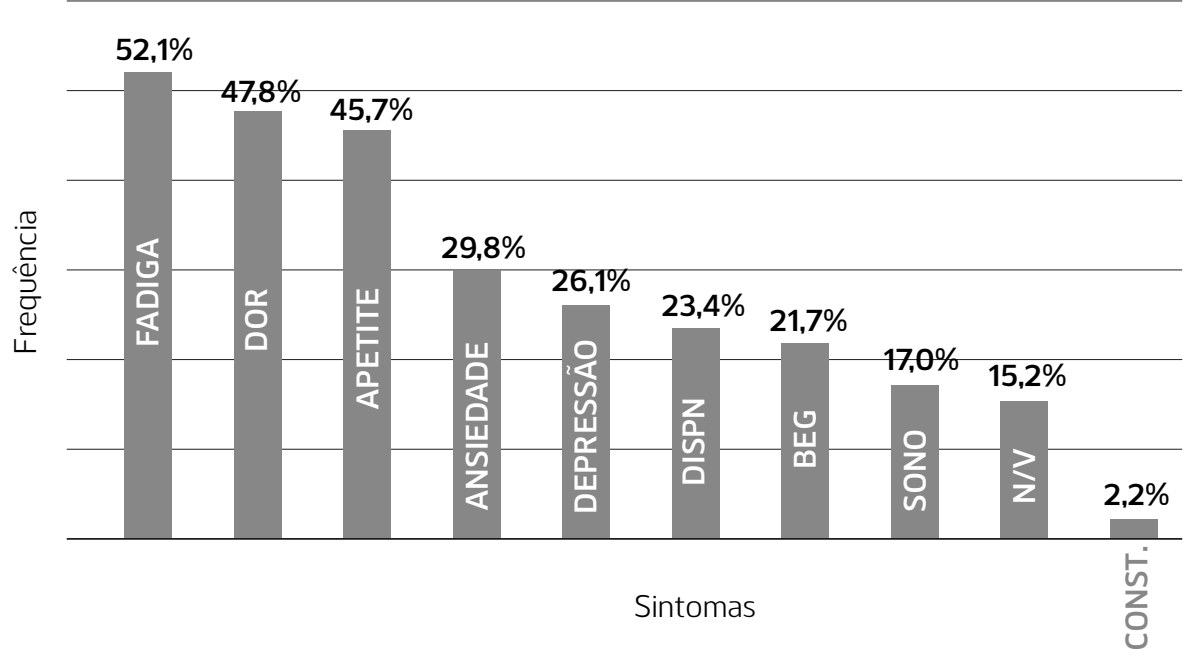

Fonte: os autores (2015)

Gráfico 2. Prevalência dos sintomas de acordo com a intensidade média, SADP, Hospital de Câncer de Barretos, $2014,2015$.

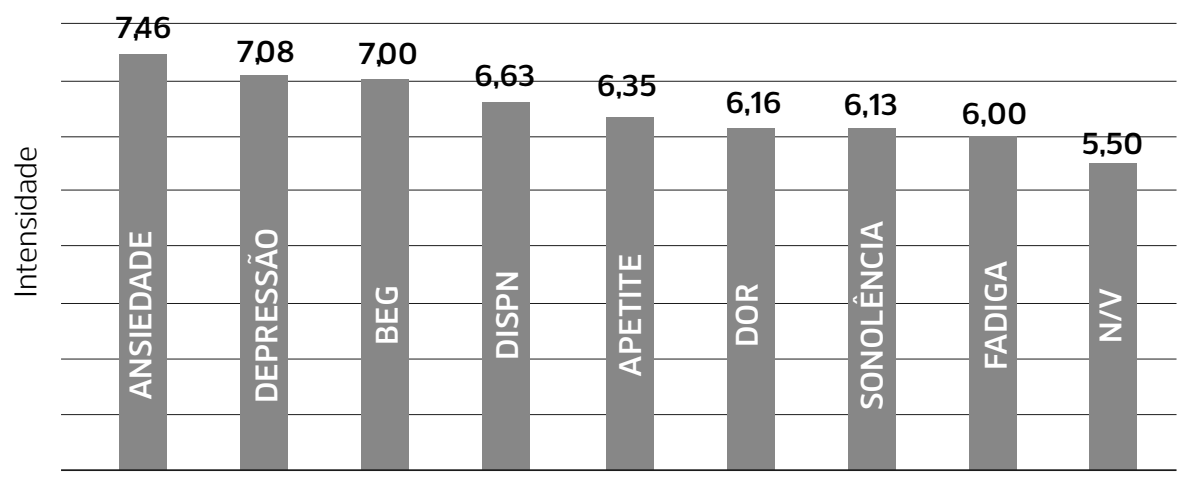

Sintomas

Fonte: os autores (2015)

\section{DISCUSSÃO}

O atendimento domiciliar vem se expandido no Brasil e, com ele, a discussão sobre alguns tópicos relevantes. O óbito no domicílio, a interface com a urgência e emergência e a interface com atenção básica são alguns desses tópicos. Paralelamente, os debates e discussões em torno do tema dos cuidados paliativos vêm se ampliando largamente. No entanto, alguns indicadores mundiais têm apontado que a situação da qualidade de morte ainda é muito ruim no território brasileiro. O ranking mundial de qualidade de morte 
nos coloca na $42^{\mathrm{a}}$ posição entre 80 países $^{33}$. O mapeamento mundial dos cuidados paliativos publicado pela Worldwide Palliative Care Alliance classifica o Brasil no nível III-b, ou seja, provisão isolada de cuidados paliativos $^{34}$ e o consumo mundial de morfina medido pelo International Narcotic Control Board aponta que o Brasil ainda consome opioides na casa das centenas de S-DDD per capita dia, enquanto que os países desenvolvidos o fazem na casa das milhares de unidades de S-DDD per capita dia ${ }^{35}$.

São $7^{19}$ os grupos de indicadores sugeridos em uma publicação de 2014 para avaliar qualidade de cuidados paliativos oferecidos em câncer:

1. Acesso aos cuidados paliativos [1a. acesso e disponibilidade; 1b. cuidado fora de hora (plantão); 1c. continuidade do cuidado];

2. infraestrutura;

3. instrumentos de avaliação;

4. pessoal [4a. equipe; 4b. compartilhar informação];

5. documentação de dados clínicos [5a. registros de dados clínicos; 5b. documentação em tempo oportuno]:

6. qualidade;

7. educação.

Classicamente, o cuidado em saúde tem sido avaliado de acordo com os critérios estabelecidos por Donabedian, a saber ${ }^{36}$ : estrutura, processo e resultado. Importante destacar que, de acordo com a estrutura disponível e como os processos estão estabelecidos e definidos, diferentes resultados serão produzidos e não necessariamente um será melhor que o outro. Como indicador de qualidade de cuidados de final de vida para pacientes com câncer avançado, internações hospitalares e procura por setor de urgência e emergência são dois indicadores de um conjunto amplo. A forma como o cuidado paliativo é levado à população é bastante diversa e adequada a cada realidade de cada país ${ }^{34}$. Por isso, o desenvolvimento de indicadores deve ser igualmente adaptado a cada região e a cada situação. Uma revisão sistematizada mostrou que a probabilidade da morte ocorrer em casa é tanto maior quanto a presença de equipe de atendimento domiciliar, como também a intensidade das visitas recebidas - quanto mais visitas, maior a probabilidade. A preferência expressa pelo paciente pelo local de morte também é um fator que favorece a morte no domicílio ${ }^{10}$. Nosso estudo mostrou que houve 50 óbitos no período estudado, sendo que 59,7\% deles ocorreram na unidade de cuidados paliativos ou no hospital da origem, seguindo a tendência brasileira ${ }^{13,14,37}$.

Um dos fatores que pode explicar esse fenômeno é o fato de que a unidade de cuidados paliativos oferece instalações extremamente confortáveis para pacientes e suas famílias, o que muitos pacientes não têm em sua própria casa. Além disso, a existência de uma equipe hospitalar bem treinada em cuidados paliativos faz com que paciente e família se sintam protegidos e acolhidos em uma fase da vida muito delicada por natureza, havendo por isso uma preferência em permanecer no hospital/unidade de cuidados paliativos. Como foi concluido em um estudo ${ }^{10}$, quanto mais visitas recebidas, maior a chance de que o óbito venha a ocorrer em casa. Embora ainda não esteja cientificamente comprovado, podemos deduzir que, de fato, um maior número de visitas ao domicílio, além de provar a disponibilidade da equipe de atendimento domiciliar, aos poucos fortalece o vínculo entre família e equipe, e aumenta a confiança na equipe, o que torna as orientações mais facilmente aceitáveis. Na população estudada aqui, não foi possível demonstrar essa correlação, provavelmente pelo tamanho amostral pequeno que se apresentou. Outra possível explicação reside no fato de que é extremamente difícil manter constante um alto número de visitas para todos os 
pacientes cadastrados no SAD/CP, uma vez que a área de abrangência da equipe é bastante ampla, com as distâncias entre os extremos da região podendo chegar a mais de 100 quilômetros. No entanto, os pacientes que morreram no domicílio receberam mais visitas da equipe do SAD/CP e compareceram menos ao setor de urgência e emergência da UCP quando comparados com aqueles que morreram na UCP ou no hospital da origem.

Na população estudada, os pacientes que tinham KPS > 40 e um PPI $<4$ tiveram uma sobrevida significativamente maior que aqueles que tinham KPS <40 e um PPI > 4. Embora esse dado seja óbvio, pois pacientes com KPS > 40 e PPI > 4 têm expectativa de sobrevida maior, ele corrobora o uso de índices como KPS e PPI para auxiliar a equipe, paciente e família a planejar melhor o cuidado a ser empregado no final da vida.

Os três sintomas mais prevalentes que os pacientes apresentaram na admissão no SAD/CP foram fadiga, dor e perda do apetite, com frequências respectivas de 52,1\%, 47,8\% e 45,7\%. Porém, os três sintomas que apresentaram maior intensidade foram ansiedade com intensidade de 7,46 e frequência de 29,8\%, depressão com intensidade de 7,08 e frequência de $26,1 \%$ e sensação de bem-estar geral com intensidade de 7,00 (muito mal-estar) e frequência de 21,7\%. Isso significa que os sintomas que apresentaram maior intensidade (em torno de 7 pontos pela ESAS, em média) tiveram uma frequência abaixo de 30\%. Esse fenômeno pode ser explicado pelo fato dos pacientes terem passado por pelo menos 1 consulta ambulatorial e tido pelo menos 1 internação na UCP, o que permitiu controlar os sintomas, exceto pela fadiga e anorexia, cuja intensidade é comum aumentar no final da vida 38,39 .

Este estudo apresenta algumas fraquezas. A primeira reside no fato de que, por se tratar do primeiro ano de atendimento do SAD/CP da UCP, tivemos apenas 72 pacientes incluídos, o que torna o tamanho amostral substancialmente pequeno dada a necessidade de categorizações realizadas e a dificuldade técnica de se produzir dados estatísticos mais confiáveis. A segunda se pauta pela qualidade de registro dos dados, como se pode notar pelas tabelas apresentadas, nas quais dados deixaram de ser registrados em muitos pacientes. Outro fator que traz fragilidade ao estudo é o fato de ser retrospectivo. Além disso, os dados foram coletados pela própria equipe de pesquisa, o que traz viés de coleta e de análise para o estudo. No entanto, percebemos que os dados trazidos aqui podem suscitar discussões no sentido de se criar indicadores de estrutura, processos e resultados que auxiliem equipes e tomadores de decisão a buscar por padrões mais altos de prestação de serviços em cuidados paliativos em domicílio.

\section{CONCLUSÃO}

Tem havido um aumento no número de serviços de cuidados paliativos, sem, no entanto, haver uma elaboração mais sistematizada da produção de indicadores que meçam estruturas, processos e resultados de ambas políticas de saúde.

Vários trabalhos têm colocado o óbito no domicílio como um indicador de qualidade de um serviço de cuidados paliativos. Na nossa observação, os pacientes que morreram no domicílio receberam mais visitas da equipe e compareceram menos ao setor de emergência oncológica paliativa, consonante com resultados de outros estudos. No entanto, essa proposição deve ser vista com cautela, pois o cuidado prestado na casa e o óbito nesse ambiente podem trazer sobrecarga física, psíquica, social e espiritual para os cuidadores familiares. 
Neste estudo, os sintomas mais prevalentes foram fadiga, dor e anorexia e os de maior intensidade foram a ansiedade, depressão e sensação de bem-estar geral reduzida, detectados na primeira visita ao domicílio.

Um estudo mais amplo, com mais indivíduos e durante um período de tempo maior, é necessário para tentar obter dados mais consistentes. Igualmente, estudos em outras regiões e municípios do País são necessários para compararmos estruturas, processos de funcionamento e resultados alcançados por tais equipes com a finalidade de gerarmos dados brasileiros mais consistentes.

Existe a necessidade do desenvolvimento/uso de indicadores específicos para equipes de cuidados paliativos, especificamente para os serviços de atendimento domiciliar, mas que se adaptem à realidade de cada instituição a fim de se evitar injustiças ou más interpretações sobre o desempenho de cada equipe de cuidados paliativos. Sugere-se para tanto, como referência, alguns trabalhos já desenvolvidos na Europa sobre o tema.

\section{REFERÊNCIAS}

1. Woitha K, Van Beek K, Ahmed N, Hasselaar J, Mollard JM, Colombet I, et al. Development of a set of process and structure indicators for palliative care: the Europall project. BMC Health Serv Res. 2012:12:381.

2. Brasil. Portaria GM 963. In: Saúde Md, editor. Brasília: Diário Oficial da União - Official Union Diary. 2013:30-2.

3. Fischer S, Min SJ, Cervantes L, Kutner J. Where do you want to spend your last days of life? Low concordance between preferred and actual site of death among hospitalized adults. J Hosp Med. 2013;8(4):178-83.

4. Aoun SM, Skett K. A longitudinal study of end-of-life preferences of terminally-ill people who live alone. Health Soc Care Community 2013:21(5):530-5

5. Ishikawa Y, Fukui S, Saito T, Fujita J, Watanabe M, Yoshiuchi K. Family preference for place of death mediates the relationship between patient preference and actual place of death: a nationwide retrospective cross-sectional study. PloS one. 2013:8(3):e56848.

6. Beccaro M, Costantini M, Giorgi Rossi P. Miccinesi G, Grimaldi M, Bruzzi P, et al. Actual and preferred place of death of cancer patients. Results from the Italian survey of the dying of cancer (ISDOC). J Epidemiology Community Health. 2006;60(5):412-6.

7. Holdsworth L, Fisher S. A retrospective analysis of preferred and actual place of death for hospice patients. Int J Palliat Nurs. 2010;16(9):424-430.

8. De Roo ML, Miccinesi G, Onwuteaka-Philipsen BD, Van Den Noortgate N, Van den Block L, Bonacchi A, et al. Actual and preferred place of death of home-dwelling patients in four European countries: making sense of quality indicators. PloS one. 2014;9(4):e93762.

9. Higginson IJ, Sarmento VP. Calanzani N. Benalia H, Gomes B. Dying at home - is it better: a narrative appraisal of the state of the science. J Palliat Med. 2013:27(10):918-924.

10. Gomes B, Higginson IJ. Factors influencing death at home in terminally ill patients with cancer: systematic review. BMJ. 2006;332(7540):51521.

11. Payne S, Brearley S, Milligan C, Seamark D. Thomas C, Wang X, et al. The perspectives of bereaved family carers on dying at home: the study protocol of 'unpacking the home: family carers' reflections on dying at home. BMC Palliat Care. 2012;11:23.

12. Delalibera M, Presa J, Barbosa A, Leal I. Burden of caregiving and its repercussions on caregivers of end-of-life patients: a systematic review of the literature. Cien Saude Colet. 2015:20(9):2731-47.

13. Marcucci FC, Cabrera MA. Death in hospital and at home: population and health policy influences in Londrina, State of Parana, Brazil (1996-2010). Cien Saude Colet. 2015;20(3):833-40

14. Telarolli Junior R, Loffredo L de C. Mortality of the elderly in a municipality of southeastern Brazil from 2006 to 2011. Cien Saude Colet. 2014:19(3):975-84.

15. Murakami N, Tanabe K, Morita T, Kadoya S, Shimada M, Ishiguro K, et al. Going back to home to die: does it make a difference to patient survival? BMC Palliat Care. 2015:14:7. 
16. Brasil. Portaria GM 825 - Atenção Domiciliar. In: Ministro Gd, editor. Brasília: DOU: 2013.

17. Pinto HA. Melhor em Casa - Caderno de Atenção Domiciliar. In: Básica DdA, editor. Brasilia: Ministério da Saúde; 2013.

18. Brasil. Manual de Monitoramento e Avaliação - Programa Melhor em Casa. In: Básica DdA, editor. Brasília: Ministério da Saúde. $2014 ; 2$.

19. Van Riet Paap J, Vernooij-Dassen M, Droes RM, Radbruch L, Vissers K, Engels Y, et al. Consensus on quality indicators to assess the organisation of palliative cancer and dementia care applicable across national healthcare systems and selected by international experts. BMC Health Serv Res. 2014;14:396.

20. Pasman HR, Brandt HE, Deliens L, Francke AL. Quality indicators for palliative care: a systematic review. J Pain Symptom Management 2009:38(1):145-56.

21. Pastrana T, Radbruch L, Nauck F. Hover G. Fegg M. Pestinger M, et al. Outcome indicators in palliative care--how to assess quality and success. Focus group and nominal group technique in Germany. Support Care Canc. 2010:18(7):859-68.

22. Dy SM, Kiley KB, Ast K, Lupu D, Norton SA, McMillan SC, et al. Measuring what matters: top-ranked quality indicators for hospice and palliative care from the american academy of hospice and palliative medicine and hospice and palliative nurses association. J Pain Symptom Management. 2015;49(4):773-81

23. Kamal AH, Gradison M, Maguire JM, Taylor D, Abernethy AP. Quality measures for palliative care in patients with cancer: a systematic review. J Oncology Pract. 2014:10(4):281-7.

24. Bruera E, Kuehn N. Miller MJ, Selmser P, Macmillan K. The Edmonton Symptom Assessment System (ESAS): a simple method for the assessment of palliative care patients. J Palliat Care. 1991;7(2):6-9.

25. Monteiro DR, Almeida MA, Kruse MHL. Translation and cross-cultural adaptation of the Edmonton Symptom Assessment System for use in Palliative Care. Rev Gaucha Enferm. 2013:34(2):163-71.

26. Timmermann C. 'Just give me the best quality of life questionnaire': the Karnofsky scale and the history of quality of life measurements in cancer trials. Chronic IIIn. 2013:9(3):179-90.

27. Morita T, Tsunoda J, Inoue S, Chihara S. The Palliative Prognostic Index: a scoring system for survival prediction of terminally ill cancer patients. Support Care Cancer. 1999;7(3):128-33.

28. Morita T, Tsunoda J, Inoue S, Chihara S. Improved accuracy of physicians' survival prediction for terminally ill cancer patients using the Palliative Prognostic Index. J Palliat Med. 2001;15(5):419-24.

29. Brasil. Melhor em Casa - Caderno de Atenção Domiciliar. In: Saúde. editor. Brasília: Editora do Ministério da Saúde. 2013.

30. Fripp JC FL, Silva SM. Caracterização de um programa de internação domiciliar e cuidados paliativos no município de Pelotas, estado do Rio Grande do Sul, Brasil: uma contribuição à atenção integral aos usuários com câncer no Sistema Único de Saúde, SUS. Epidemiol Serv Saúde. 2012:21(1):69-78.

31. Abedini NC, Hechtman RK, Singh AD, Khateeb R, Mann J, Townsend W, et al. Interventions to reduce aggressive care at end of life among patients with cancer: a systematic review. Lancet Oncol. 2019;20(11):e627-e36.

32. Earle CC, Park ER, Lai B, Weeks JC, Ayanian JZ, Block S. Identifying potential indicators of the quality of end-of-life cancer care from administrative data. J Clin Oncol. 2003:21(6):1133-8.

33. Unit El. The 2015 Quality of Death Index - Ranking Palliative Care Around the World. 2015

34. WHO. Global Atlas of Palliative Care. London: Worldwide Palliative Care Alliance; 2014.

35.WHO. Availability of Internationally Controlled Drugs - Ensuring Adequate Access for Medical Purposes. Viena: INCB; 2015.

36.Donabedian A. Evaluating the quality of medical care. 1966. Milbank Q. 2005:83(4):691-729.

37.Silva KLS, R.; Leite, J.C.A.; Seixas, C.T.; Gonçalves, A.M. Home Care in the Brazilian National Health. Rev Saúde Pública. 2005;39(3):1-6.

38.Yennurajalingam S. Fatigue and asthenia. Oxford: Oxford University Press; 2015

39.Childs DS, Jatoi A. A hunger for hunger: a review of palliative therapies for cancer-associated anorexia. Ann Palliat Med. 2019;8(1):50-8. 